\title{
Pulmonary function testing precautions in a time of COVID-19
}

\section{Posted April 13, 2020}

\section{ABSTRACT}

During the COVID-19 pandemic, Cleveland Clinic has put in place several pulmonary function testing precautions to reduce the risk of infection. Precautions include efforts to perform only essential testing, wear personal protective equipment, reduce exposure to aerosolized particles, manage virtually by phone conference, conserve scare medications, clean equipment as recommended, consolidate testing, and defer testing.

\section{PFT AND COVID-19}

During the COVID-19 pandemic, Cleveland Clinic's pulmonary function testing (PFT) laboratories have put several initiatives in place to do our part in flattening the curve of infection.

- Perform only essential testing. Most of this testing is in candidates for lung transplant, patients undergoing surveillance for side effects of chemotherapy, surgical candidates, and patients with chronic illnesses for which PFTs are needed for evaluation or in triaging treatment (eg, interstitial lung disease, pulmonary arterial hypertension).

- Wear personal protective equipment (PPE). All technicians must wear a surgical mask, face shield or goggles, and gloves during testing. If any tests require aerosolization of any medication, the technician should wear complete PPE: a gown, gloves, face shield or goggles, and an N95 respirator, a powered air purifying respirator, or both. Once the patient has been started on the treatment, the technician should wait outside of the room and periodically check in until the treatment is completed.

The statements and opinions expressed in COVID-19 Curbside Consults are based on experience and the available literature as of the date posted. While we try to regularly update this content, any offered recommendations cannot be substituted for the clinical judgment of clinicians caring for individual patients.

doi:10.3949/ccjm.87a.ccc006
- Manage virtually, if possible by working with clinicians to identify which patients can be managed virtually or by phone conference, and reschedule their PFT tests for a later date.

- Reduce exposures to aerosolized particles. We have suspended methacholine challenge tests, sputum induction, and aerosol treatments, and have limited pentamidine treatments to fewer locations.

- Conserve scarce medications. Short-acting beta-agonist (albuterol) inhalers, used to evaluate bronchodilation, are currently in short supply. To conserve inhalers, we have changed our practice of delivering 4 puffs via a valve-holding chamber (spacer) as recommended by the American Thoracic Society and European Respiratory Society guidelines ${ }^{1}$ to delivering only 2 puffs. Also, even if the order says "spirometry before and after bronchodilator," if the patient has undergone previous testing that showed no change after receiving the medication, we withhold the bronchodilator unless the clinician feels it is necessary.

- Keep to current recommendations for cleaning equipment. We use Super Sani-Cloth (purple top) germicidal disposable wipes (PDI, Woodcliff Lake, NJ) for hard surfaces and Sani-Cloth AF3 (gray top) for glass and other clear surfaces. The manufacturer of the MicroGard II filter (Vyaire Medical, Hoechberg, Germany), used in pulmonary function testing, states the COVID-19 particles are too large to pass through their filter (communication, Juergen Reinstaedtler and Waldemar Fabry, March 18, 2020), based on testing by Nelson Labs. ${ }^{2}$

- Consolidate testing. Due to the current low volume of patients, we have consolidated our testing down from 24 laboratory locations to only 5 . This has allowed us to redeploy staff to cross-train for inpatient care throughout the Ohio locations to prepare for a surge in hospital admissions, should this happen.

- Defer testing. As we continue to prepare for the COVID-19 projected surge, we have asked for patients not to be rescheduled until August at the earliest. 


\section{REFERENCES}

1. Miller MR, Hankinson J, Brusasco V, et al; ATS/ERS Task Force. Standardisation of spirometry. Eur Respir J 2005; 26(2):319-338. doi:10.1 183/09031936.05.00034805
2. Nelson Labs. Bacterial \& viral filtration efficiency (BFE/VFE). https:// www.nelsonlabs.com/testing/bacterial-viral-filtration-efficiencybfe-vfe/ Accessed April 14, 2020. 